\title{
Increasing WSN Lifetime using Clustering and Fault Tolerance Methods
}

Sama Hussam Sabah *, Muayad Sadik Croock

Computer Engineering Department, University of Technology, Baghdad, Iraq

\author{
Correspondence \\ *Sama Hussam Sabah \\ Computer Engineering Department, \\ University of Technology, Baghdad, Iraq \\ Email: 120634@student.uotechnology.edu.iq
}

\begin{abstract}
Energy consumption problems in wireless sensor networks are an essential aspect of our days where advances have been made in the sizes of sensors and batteries, which are almost very small to be placed in the patient's body for remote monitoring. These sensors have inadequate resources, such as battery power that is difficult to replace or recharge. Therefore, researchers should be concerned with the area of saving and controlling the quantities of energy consumption by these sensors efficiently to keep it as long as possible and increase its lifetime. In this paper energy-efficient and fault-tolerance strategy is proposed by adopting the fault tolerance technique by using the self-checking process and sleep scheduling mechanism for avoiding the faults that may cause an increase in power consumption as well as energy-efficient at the whole network. this is done by improving the LEACH protocol by adding these proposed strategies to it. Simulation results show that the recommended method has higher efficiency than the LEACH protocol in power consumption also can prolong the network lifetime. In addition, it can detect and recover potential errors that consume high energy.
\end{abstract}

KEYWORDS: Fault-Tolerance, LEACH, Sleep-Scheduling Mechanism, Self-checking process, WSN.

\section{INTRODUCTION}

In most all critical applications, such as environmental monitoring, health care monitoring, Wireless Sensor Networks (WSN) are used [1]. The sensor is the basic unit of WSN because of its essential role in sensing and gathering information from the monitoring areas but it has restricted resources like energy [2] and [3]. This is due to its batterypowered which is difficult to be recharged or replaced [4], Thus energy is the most critical factor to the conservation of the survival in these sensors [5]. In direct communications, sensors send the data that is sensed to the sink or base station regardless of the distance between them which causes consumption of high energy in the transmission medium. This leads to energy dissipation early [6] and [7], but WSN depends on the clustering mode of the monitored area separated into clusters [8]. Each cluster has its own member nodes and cluster head node that is responsible for communication inside the cluster and forwards gathered data from nodes outside the cluster [9] to eliminate the long distances between nodes which leads to energy conservation for a longer period of time, and the first protocol designed to adopt the principle of clustering is LEACH protocol [10]. Which still suffers from some deficiencies that can be looked at and addressed in this paper.
Also, these individual sensor nodes make WSN more fault-prone cause of resource constraints [11], and this effect directly to energy rates and the release of the batteries earlier [12]. Therefore, we must take care of controlling these errors and addressing them to save available energy. therefore, a fault tolerance system is required to achieve that goal [13]. To increasing system lifetime, numerous routing algorithms be created in such a manner [14]and [15] as we will mentioned in related works part. Out of all these, the clustering algorithm has increased a great deal of significance in expanding the system lifetime; consequently, the effectiveness of the sensors in it [16]. Grouping gives a powerful method to Extend wireless sensor network lifetime [17].

In this paper, we introduce a method that can increase network lifetime depends on two enhanced algorithms. The first is to make the use of available energy more efficiently as possible by enhancing the leach protocol by adding an active selection of member nodes depend on distances between them. Second is the fault tolerance algorithm adding inside each sensor node depending on two steps (detect and recovery) from faults that make the system consume more power. This is done using the self-checking process in each sensor node. the rest of this paper is divided into 5 section 2 explain the related works in reducing power consumption and fault tolerance in WSN, section 3 shows the proposed

This is an open access article under the terms of the Creative Commons Attribution License, which permits use, distribution and reproduction in any medium, provided the original work is properly cited.

(C) 2021 The Authors. Published by Iraqi Journal for Electrical and Electronic Engineering by College of Engineering, University of Basrah. 
algorithms in flowcharts and explain their operation steps, section 4 shows the results compared with LEACH protocol depend on two factors (energy consumed, lifetime) of the network, section5 explaining conclusions of this work.

Related Works: Reducing power consumption in a WSN is considered a major interest in recent decades. Consequently, many algorithms have been proposed for increasing the WSN lifetime and conservation of the available energy.

In [18], the authors introduced a method to increase network lifetime by making enhancing in leach protocol by fixing one's leach disadvantage that it is not taking the distance from cluster head to Data collection station in consideration. They could decrease energy consumption about 0.7 joule, but they have not taken fault tolerance in the consideration. In [19], the authors made enhance in leach protocol and take a residual energy at each node in the consideration as well as the distance from cluster head to a data collection station. While in [20], the authors developed a hybrid energy-efficient distributed algorithm in the fault tolerance stage by making the decision about faults occur in the base station.

This is to do appropriate action and isolated faulty nodes and take its value by applying weighted median method to increase the corrected data rate. Because of the importance of fault-tolerance to increases the system's reliability [21], the researchers collected different and modern types of faults and discussed the pros and cons of each type and the appropriate solutions for each type of faults to achieve higher information trust. In [22], the researchers designed a routing protocol that achieves the saving power of the network depending on remaining energy of each node, whereby the information is sent from the sender to the base station. It checks the neighboring nodes to allocate the one with the highest residual energy, through which the information is transmitted.

This can achieve a balance in the available power of a network and increasing network lifetime. The researchers in [23] worked on organizing the transceiver device's operation using sleep scheduling to control energy exchange efficiently, using Zigbee protocol because it has the advantage of avoiding collisions that lead to waste of energy.

In [24], the authors used k-means algorithm for path creation and backpropagation algorithm to optimize this path, and they got a decrease in power consumption of about 6.3 joule with optimization. Still, they did not take the path between nodes and base station in consideration. In [25], the authors made develop in grey wolf optimization that it works on cluster head. Still, it is very complex and takes a long time to make its calculation the authors made it faster to compute distance and very simple and achieve high lifetime in the network.

\section{PROposed SySTEM}

As described above, the proposed method is built based on two concepts that can reduce the consumed power in an efficient way. These concepts are enhanced clustering method and power fault detection. "Fig. 1" explain the structure of the proposed system in three steps: 1. Clustering the WSN using LEACH protocol, 2. Enhancing LEACH protocol by adding a new mechanism to it (active selection of member nodes depend on distances), 3. Adding fault tolerance technique by using self-checking process inside each sensor node.

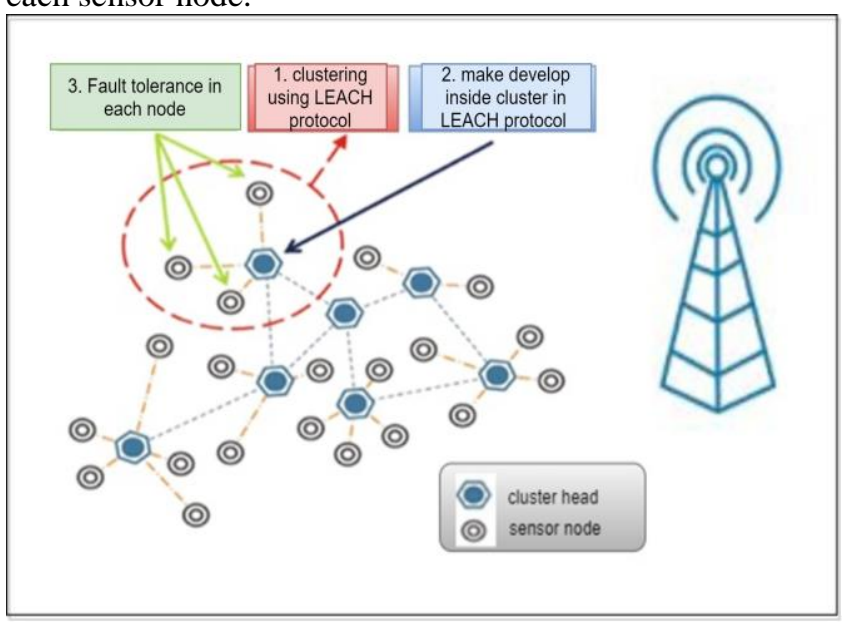

Fig.1: The Structure of the system

From this figure, we note that the WSN contains the base station and different sensors, spread randomly in the area that we want to monitor. In the beginning, the sensors start to connecting together and recognizing the neighbors. In case the sensors send their readings direct to the base station, the energy consumed quickly and the nodes can die early, in which the whole network is dead. Therefore, the main issue is how to control the communication between these sensors to make the lifetime of the system longer. To decrease the amount of consumed power, the leach protocol is considered to develop culturing as show in "Fig. 2".

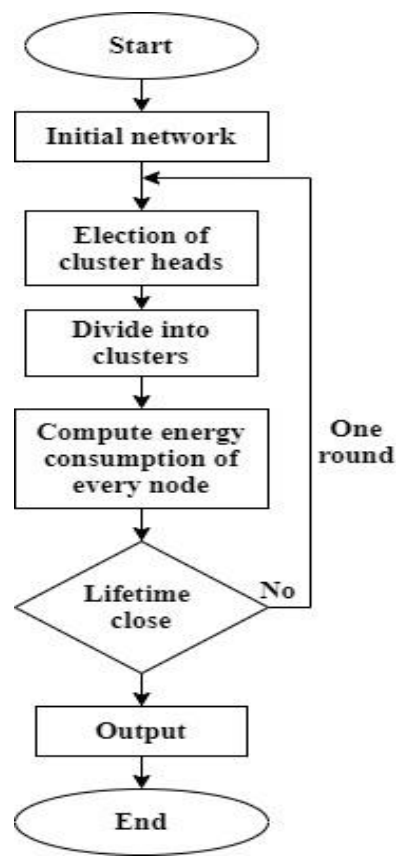

Fig.2: Flowchart of LEACH protocol to make Clustering in WSN [10]. 
As shown in the flowchart of LEACH protocol cluster head being to select from member nodes by using equation (1) and then remaining member node decides to join cluster depend on strong signal broadcasting from elected cluster heads to configure clusters, then compute energy consumption of every node by using simple first order radio model as shown in Fig. 3

$$
T(n)= \begin{cases}\frac{p}{1-p\left(r \bmod \frac{1}{p}\right)}, & \text { if } n \in G \\ 0 & \text { otherwise }\end{cases}
$$

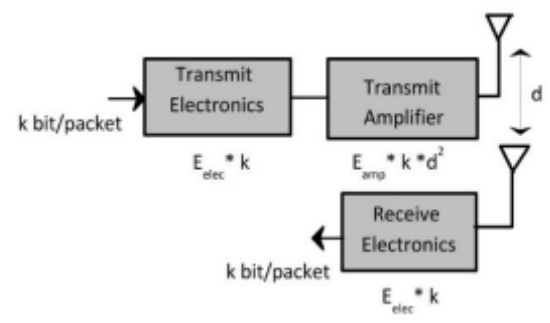

Energy consumption for transmitter is:

$$
E_{T x}(K, d)=\left\{\begin{array}{c}
E_{\text {elec }} * K+K * \varepsilon_{f s} * d^{2}, d \leq d_{0} \\
E_{\text {elec }} * K+K * \varepsilon_{m p} * d^{4}, d>d_{0}
\end{array}\right.
$$

Energy consumption for receiver is:

$$
E_{R x}=E_{\text {elec }} * K
$$

Fig.3 Simple energy radio model and its equations to compute require energy to transmit and receive depend on distance d [10].

Finally, LEACH still to work until all nodes become die some recorded weaknesses in the LEACH protocol must point out, it does not take the nearest distances between member nodes into consideration. In this paper, the proposed algorithm addresses this weakness by taking it into consideration. leach protocol approves in the WSN, the leach protocol assumes the initial energy for each node is equal and then accomplishes a new algorithm in leach protocol Based on scheduling operations of member nodes in each cluster, by an active selection of member nodes first the distance " $D$ " between node and cluster head compute by using equation (2) and then the distance between the same node and its neighbors " $\mathrm{d}$ " also by using equation (2) then compare between results to make nodes that nearest to each other, turn-on one node and others go to sleep mode rather than two nodes take the same reading about the monitoring area let one operate and another go to sleep mode as shown in the flowchart "Fig. 4". This can support the reduction of more energy consumption to prolong its system lifetime.

$$
d=\sqrt{\left(X_{2}-X_{1}\right)^{2}+\left(Y_{2}-Y_{1}\right)^{2}}
$$

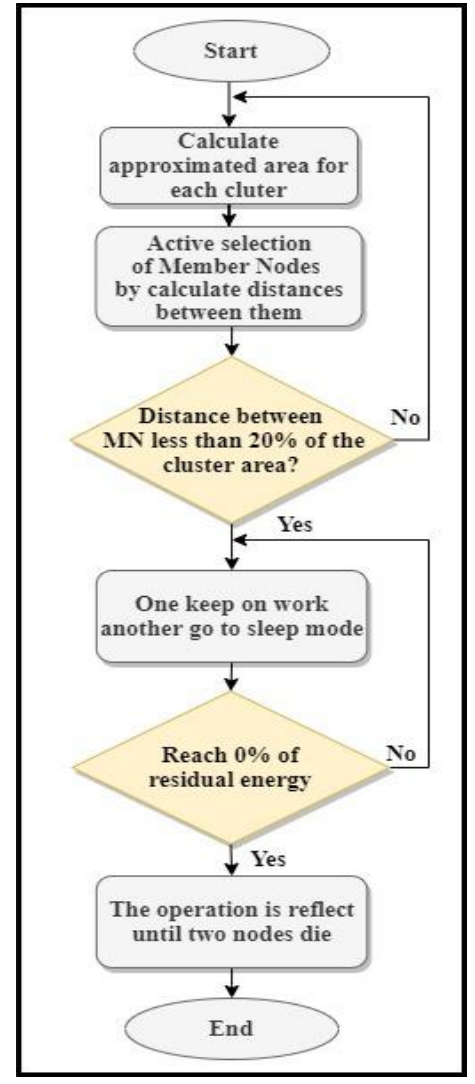

Fig.4: .Enhanced Clustering in LEACH protocol.

The following steps guide you through the algorithm.:

1. Calculating each cluster's estimated area by using equation (2).

2. Calculating the area covered by Member Nodes (MN). In case the area is less than $20 \%$ of the cluster area, one of these Neighbor nodes is turned to sleep mode.

3 . This is repeated until the active node has consumed its energy, at which point the sleep node is awakened to control the monitoring job of the wanted area.

4. This procedure is repeated until two nodes have died. (Depleted Of energy).

After this, the self-checking process is performed in each sensor node as a fault-tolerance method to increase its lifetime. The algorithm puts a threshold value of maximum required energy to transmit and receive data, each node monitors and checks its power consumption behavior and takes proper action for saving available node energy.

The proposed fault tolerance method deals with each detected fault in two steps: fault detection and fault recovery. It detects the power fault using a self-checking process by found the transmission needs more than the maximum threshold and takes action as a power recovery by setting the underlying node to sleep mode until two round and then restart it to normal mode if the same node detects the fault another then the maintenance team should be alarm because the fault may be damage in hardware, not software. "Fig. 5" shows the proposed power-based self-checking process in fault detection and recovery. 


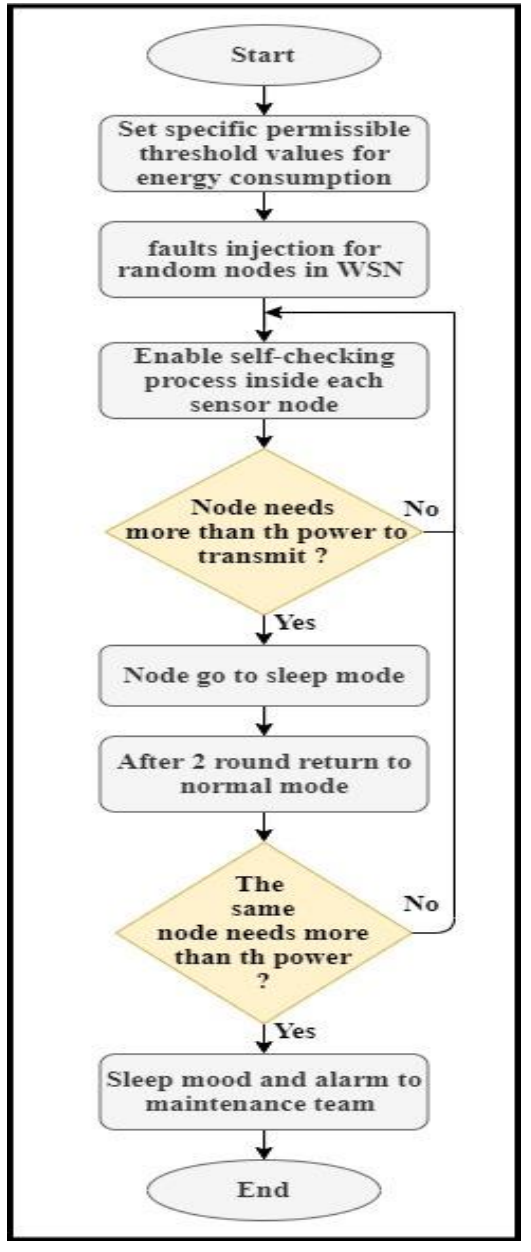

Fig.5: Proposed Self-Checking Process Algorithm.

The adopted steps of the algorithm are explained as: 1.Put a threshold for maximum energy to transmit.

2.assume the faults that wanted energy to transmit data more than the maximum threshold.

3.Checking the sensor node's consume energy, if it is more than the max threshold, the node goes to sleep mode until two rounds then go back to normal mode

4.If the same node prone to the fault the maintenance team should alarm.

\section{RESUlTS OF SIMULATIONS}

To check the efficiency of the Suggested system, a simulator to represent the proposed system was built using MATLAB. A WSN is built with 100 sensor nodes spread randomly in an area of $100 * 100$ meters. The base station is allocated in the center. Each sensor node has an initial energy 2 joule, as shown in "Fig. 6" which shows the distribution of the nodes. The cluster heads are determined depending on the evaluated distances from the base station. At the initialization, each sensor node starts to join the proper cluster for exchanging the data. Meanwhile, the selfchecking process is continuous in the nodes until all sensors are dead and the whole network would drain as shown in "Fig. 7".

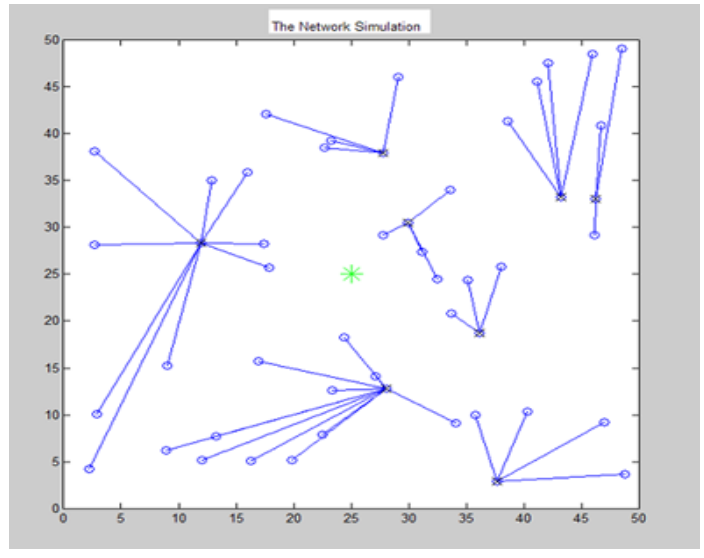

Fig.6: Start of network simulation

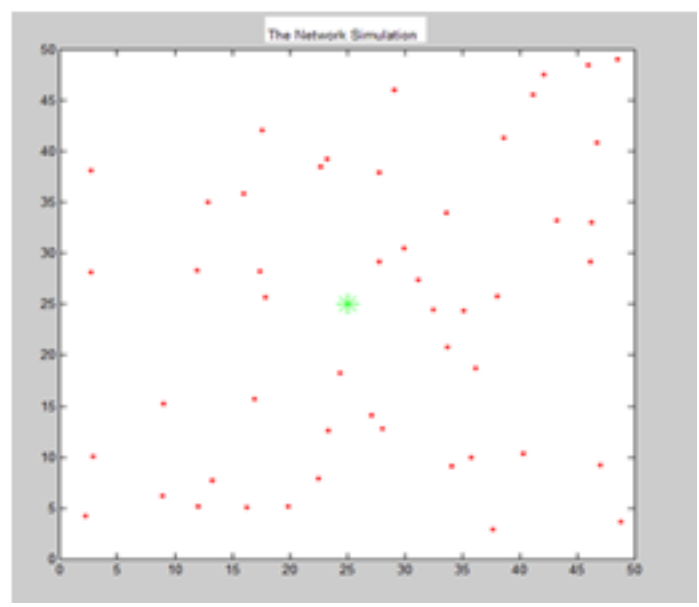

Fig.7: End of network simulation

\begin{tabular}{|cl|}
\hline$*$ & Base Station \\
0 & Sensor Node \\
& Cluster head \\
& Communication \\
\hline
\end{tabular}

TABLE I

Simulation Parameters Used In The System.

\begin{tabular}{|ll|}
\hline Parameters & Values \\
\hline Area's length & $100 \mathrm{~m}$ \\
Area's width & $100 \mathrm{~m}$ \\
x coordination of base station & Length / 2 \\
y coordination of base station & Width / 2 \\
Number of Nodes in the area & 100 \\
Each node 's initial energy & $2 \mathrm{j}$ \\
Energy for transferring each & $50 * 0.000000001$ \\
bit & \\
Energy to collect to each bit & $50 * 0.000000001$ \\
Energy of Data aggregation & $5^{*} 0.000000001$ \\
\hline
\end{tabular}

Table (1) displays simulation parameters used in our proposed model that depend on radio medium who need this value in calculation when transmitting or receiving data. The proposed method is compared with leach protocol [17] Due 
to the LEACH protocol don't take distances between nodes in cosidration also don't take fault tolerance in the consideration.

When executing the approach with 100 sensor nodes, we note that the proposed method saves more power and increases the WSN lifetime compared with the LEACH protocol.

At the start, both approaches are similar because a lot of energy consumed in the communication step at the beginning of the network.

From the result simulation note that in leach protocol the whole network would drain at 2000 iteration while the proposed algorithm with first enhance is drained at 2500 iteration and with third enhance is drain above 4500 iterations as shown in "Fig. 8" which shows a number of operational nodes per rounds in leach protocol in red line and proposed algorithm with first enhance in the green line and blue line with final enhancement.

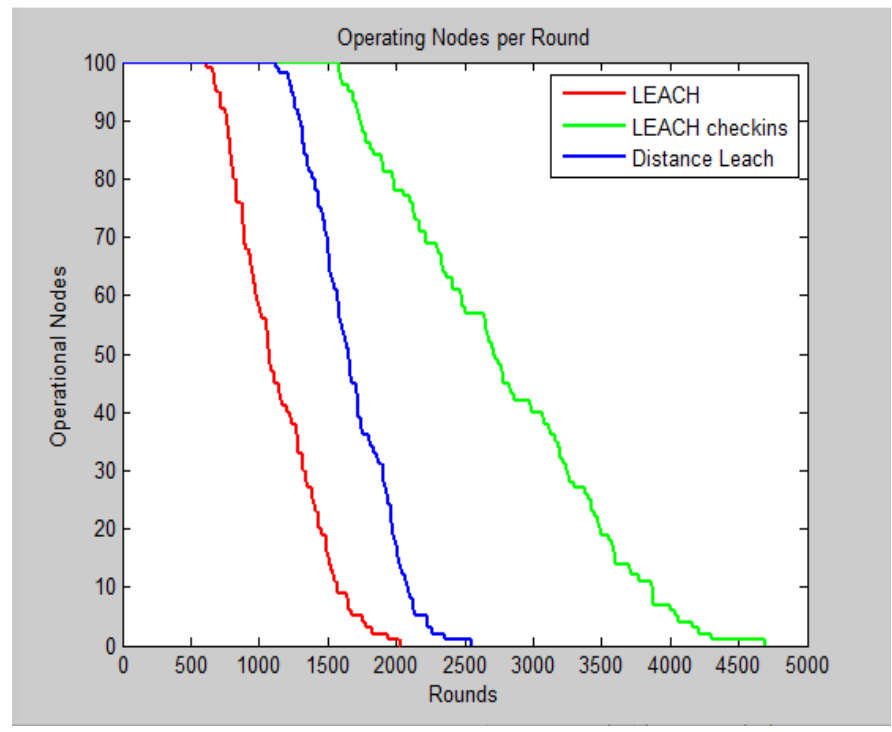

Fig.8: Comparison proposed system with leach protocol in term of alive nodes per rounds.

The Red line in the label shows the execution of standard LEACH protocol which explains in the first part in II the proposed system and, the Green line shows the execution of the first enhanced algorithm that has been explaining in flowchart Fig.4, Blue line shows the execution of second enhance algorithm that has been explained in Fig.5.

Also, the simulation make comparison of consumed energy of the whole network as show in the spectrum of the energy consumed per transmission in "Fig. 9" whole network is 200 joule supplies.

$($ Energy total $=$ No of nodes $*$ initial energy $)$

The whole network drains the available power in LEACH protocol at 1600 transmission while in 1600 transmission the proposed algorithm still saves 30 joules in first enhance and save approximately 80 joules in second enhancement.

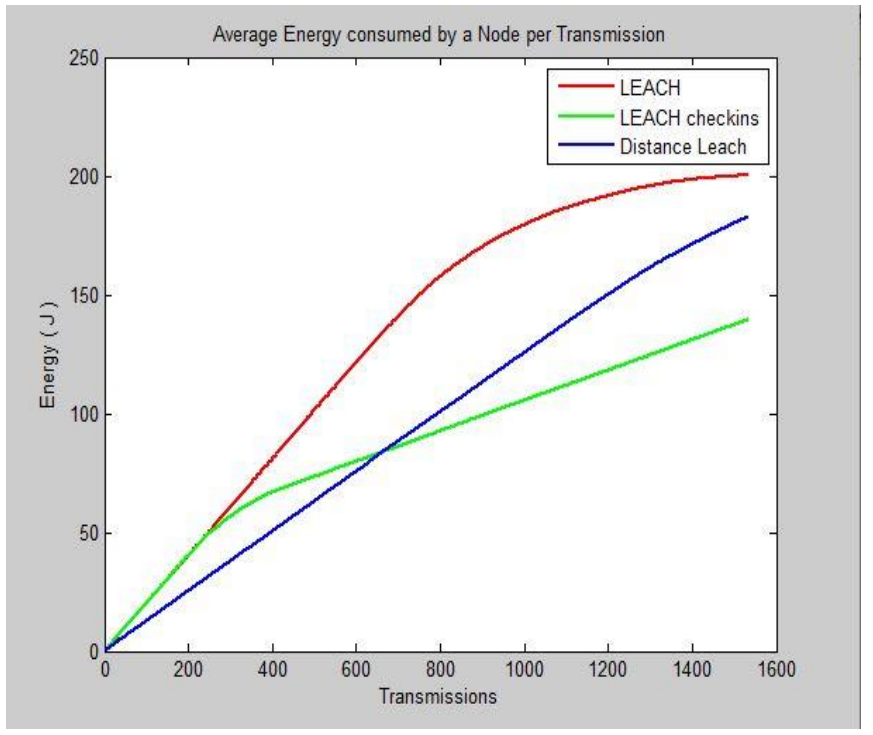

Fig.9: Comparison proposed system with leach protocol in term of Power consumption.

\section{CONCLUSIONS}

An energy-efficient method for WSN was proposed. This method adopted two concepts of power fault tolerance and enhanced leach protocol. The enhanced leach protocol considered the small distances between nodes. Moreover, the power fault tolerance method decreased the consumed power by faulty nodes. The Results obtained demonstrated superior efficiency of the proposed system lifetime and power consumption of underlying WSN compared with basic LEACH protocol.

\section{CONFLICT OF INTEREST}

The authors have no conflict of relevant interest to this article.

\section{REFERENCES}

[1] S. A. Shaikh, M. Ali, R. Ali, S. H. Memon, and N. K. Pathan, "Design of Solar Tracking System Using Piezoelectric Effect for Multi - Power Generation Based on IoT", Iraqi Journal for Electrical and Electronic Engineering, vol. 03, no. 02, pp. 30-33, 2020.

[2] E. Technology, F. Komal, T. Khanam, and Z. Ahmed, "Wireless Power Transfer for Battery Charging of Electrical Vehicles", Iraqi Journal for Electrical and Electronic Engineering, vol. 01, no. 01, pp. 19-23, 2018.

[3] S. Savitha, S. C. Lingareddy, and S. Chitnis, "Energy efficient clustering and routing optimization model for maximizing lifetime of wireless sensor network," Int. J. Electr. Comput. Eng., vol. 10, no. 5, pp. 4798-4808, 2020, doi: 10.11591/ijece.v10i5.pp4798-4808.

[4] S. A. Mohammed, K. A. E. Aly, and A. M. Ghuniem, "An enhancement process for reducing energy consumption in wireless sensor network," Int. J. Emerg. Trends Eng. Res., vol. 8, no. 6, pp. 2765-2769, 2020, doi: 10.30534/ijeter/2020/89862020. 
[5]V. Saranya, S. Shankar, and G. R. Kanagachidambaresan, "Energy Efficient Data Collection Algorithm for Mobile Wireless Sensor Network," Wirel. Pers. Commun., vol. 105, no. 1, pp. 219-232, 2019, doi: 10.1007/s11277-0186109-3.

[6] A. Ahmad, N. Javaid, Z. A. Khan, U. Qasim, and T. A. Alghamdi, "(ACH)2: Routing scheme to maximize lifetime and throughput of wireless sensor networks," IEEE Sens. J., vol. 14, no. 10, pp. 3516-3532, 2014, doi: 10.1109/JSEN.2014.2328613.

[7] D. W. Sambo, B. O. Yenke, A. Förster, and P. Dayang, "Optimized clustering algorithms for large wireless sensor networks: A review," Sensors (Switzerland), vol. 19, no. 2, 2019, doi: 10.3390/s19020322.

[8] V. Sharma, P. Rajpoot, A. Gupta, K. Dubey, N. Pandey, and N. Verma, "Heterogeneous clustering for energy optimization in wireless sensor networks," Proc. 9th Int. Conf. Cloud Comput. Data Sci. Eng. Conflu. 2019, pp. 92-99. 2019 ,

doi: 10.1109/CONFLUENCE.2019.8776933.

[9] Q. Bian, Y. Zhang, and Y. Zhao, "Research on clustering routing algorithms in wireless sensor networks," 2010 Int. Conf. Intell. Comput. Technol. Autom. ICICTA 2010, vol. 2, pp. 1110-1113, 2010, doi: 10.1109/ICICTA.2010.343.

[10] T. M. Behera, S. K. Mohapatra, U. C. Samal, M. S. Khan, M. Daneshmand, and A. H. Gandomi, "Residual energy-based cluster-head selection in WSNs for IoT application," IEEE Internet Things J., vol. 6, no. 3, pp. 5132-5139, 2019, doi: 10.1109/JIOT.2019.2897119.

[11] D. S. Park, "Fault tolerance and energy consumption scheme of a wireless sensor network," Int. J. Distrib. Sens. Networks, vol. 2013, no. March, 2013, doi: $10.1155 / 2013 / 396850$.

[12] Z. Zhang, A. Mehmood, L. Shu, Z. Huo, Y. Zhang, and M. Mukherjee, "A survey on fault diagnosis in wireless sensor networks," IEEE Access, vol. 6, pp. 11349-11364, 2018, doi: 10.1109/ACCESS.2018.2794519.

[13] P. H. and B. S. B. Karunakara Rai, J. P. Harshitha, Radhika S. Kalagudi, B. S. Priyanka Chowdary, and Texture Features of Chest X-Rays, no. January. Springer Singapore, 2019.

[14] K. Arulmozhi, V. R. S. Dhulipala, and R. Prabakaran, "A study on optimized power consumption routing in Wireless Sensor Networks," ICECT 2011 - 2011 3rd Int. Conf. Electron. Comput. Technol., vol. 3, pp. 271-274, 2011, doi: 10.1109/ICECTECH.2011.5941752.

[15] M. Abo-Zahhad, M. Farrag, A. Ali, and O. Amin, “An energy consumption model for wireless sensor networks," 5th Int. Conf. Energy Aware Comput. Syst. Appl. ICEAC 2015, 2015, doi: 10.1109/ICEAC.2015.7352200.

[16] P. C. Srinivasa Rao and H. Banka, "Energy efficient clustering algorithms for wireless sensor networks: novel chemical reaction optimization approach," Wirel. Networks, vol. 23, no. 2, pp. 433-452, 2017, doi: 10.1007/s11276-015-1156-0.

[17] M. R. Mundada, N. Thimmegowda, T. Bhuvaneswari, and V. Cyrilraj, "Clustering in wireless sensor networks: Performance comparison of EAMMH and LEACH protocols using MATLAB," Adv. Mater. Res., vol. 705, pp. 337-342, 2013, doi: 10.4028/www.scientific.net/AMR.705.337.

[18] S. Devi, I. Ahmed, M. Urvashi, and P. G. Student, "Optimization Technique to Improve the Energy Efficiency in WSN : LEACH-MGWO," no. 5, pp. 176182, 2018.

[19] Z. Arabi and R. Parikhani, "E FP : NEW E NERGY EFFICIENT F AULT - TOLERANT," vol. 4, no. 6, pp. 111-119, 2012.

[20] S. Jafarali Jassbi and E. Moridi, "Fault Tolerance and Energy Efficient Clustering Algorithm in Wireless Sensor Networks: FTEC," Wirel. Pers. Commun., vol. 107, no. 1, pp. 373-391, 2019, doi: 10.1007/s11277-01906281-6.

[21] A. Choudhary, S. Choudhary, and A. Mishra, "Review on Fault Tolerance in Wireless Sensor Network," Int. J. Comput. Appl., vol. 182, no. 45, pp. 22-25, 2019, doi: 10.5120/ijca2019918597.

[22] C. H. Lin, H. Y. Lin, and W. Bin Lee, "Routing protocols with power saving and data salvation for wireless sensor networks," Proc. - 2012 7th Int. Conf. Broadband, Wirel. Comput. Commun. Appl. BWCCA 2012, pp. 468-471, 2012, doi: 10.1109/BWCCA.2012.83.

[23] D. Goyal and Sonal, "Power management in Wireless Sensor Network," Proc. 10th INDIACom; 2016 3rd Int. Conf. Comput. Sustain. Glob. Dev. INDIACom 2016, vol. 4, no. 4, pp. 598-601, 2016.

[24] K. M. Krishnapriya, S. Anand, and S. Sinha, "A customised approach for reducing energy consumption in wireless sensor network," Int. J. Innov. Technol. Explor. Eng., vol. 8, no. 8, pp. 1427-1431, 2019.

[25] A. O. Abu Salem and N. Shudifat, "Enhanced LEACH protocol for increasing a lifetime of WSNs," Pers. Ubiquitous Comput., vol. 23, no. 5-6, pp. 901-907, 2019, doi: 10.1007/s00779-019-01205-4. 